\title{
Order Level Inventory Models for Deteriorating Seasonable/Fashionable Products with Time Dependent Demand and Shortages
}

\author{
K. Skouri ${ }^{1}$ and I. Konstantaras ${ }^{1,2}$ \\ ${ }^{1}$ Department of Mathematics, University of Ioannina, 45110 Ioannina, Greece \\ ${ }^{2}$ Hellenic Army Academy, Vari 16673, Attica, Greece \\ Correspondence should be addressed to I. Konstantaras, ikonst@cc.uoi.gr
}

Received 18 September 2008; Revised 3 July 2009; Accepted 20 July 2009

Recommended by Wei-Chiang Hong

\begin{abstract}
An order level inventory model for seasonable/fashionable products subject to a period of increasing demand followed by a period of level demand and then by a period of decreasing demand rate (three branches ramp type demand rate) is considered. The unsatisfied demand is partially backlogged with a time dependent backlogging rate. In addition, the product deteriorates with a time dependent, namely, Weibull, deterioration rate. The model is studied under the following different replenishment policies: (a) starting with no shortages and (b) starting with shortages. The optimal replenishment policy for the model is derived for both the above mentioned policies.
\end{abstract}

Copyright (C) 2009 K. Skouri and I. Konstantaras. This is an open access article distributed under the Creative Commons Attribution License, which permits unrestricted use, distribution, and reproduction in any medium, provided the original work is properly cited.

\section{Introduction}

It is observed that the life cycle of many seasonal products, over the entire time horizon, can be portrayed as a period of growth, followed by a period of relatively level demand and finishing with a period of decline. So researchers commonly use a time-varying demand pattern to reflect sales in different phases of product life cycle. Resh et al. [1] and Donaldson [2] are the first researchers who considered an inventory model with a linear trend in demand. Thereafter, numerous research works have been carried out incorporating timevarying demand patterns into inventory models. The time dependent demand patterns, mainly, used in literature are, (i) linearly time dependent and, (ii) exponentially time dependent (Dave and Patel [3], Goyal [4], Hariga [5], Hariga and Benkherouf [6], Yang et al. [7]). The time dependent demand patterns reported above are unidirectional, that is, increase continuously or decrease continuously. Hill [8] proposed a time dependent demand pattern by considering it as the combination of two different types of demand in two successive 
time periods over the entire time horizon and termed it as "ramp-type" time dependent demand pattern. Then, inventory models with ramp type demand rate also are studied by Mandal and Pal [9], Wu et al. [10] and Wu and Ouyang [11], Wu [12], Giri et al. [13], and Manna and Chaudhuri [14]. In these papers, the determination of the optimal replenishment policy requires the determination of the time point, when the inventory level falls to zero. So the following two cases should be examined: (1) this time point occurs before the point, where the demand is stabilized, and (2) this time point occurs after the point, where the demand is stabilized. Almost all of the researchers examine only the first case. Deng et al. [15] reconsidered the inventory model of Mandal and Pal [9] and $\mathrm{Wu}$ and Ouyang [11] and studied it exploring these two cases. Skouri et al. [16] extend the work of Deng et al. [15] by introducing a general ramp type demand rate and considering Weibull distributed deterioration rate.

The assumption that the goods in inventory always preserve their physical characteristics is not true in general. There are some items, which are subject to risks of breakage, deterioration, evaporation, obsolescence, and so forth. Food items, pharmaceuticals, photographic film, chemicals, and radioactivesubstances are few items in which appreciable deterioration can take place during the normal storage of the units. A model with exponentially decaying inventory was initially proposed by Ghare and Schrader [17]. Covert and Phillip [18] and Tadikamalla [19] developed an economic order quantity model with Weibull and Gamma distributed deterioration rates, respectively. Thereafter, a great deal of research efforts have been devoted to inventory models of deteriorating items, the details can be found in the review articles by Raafat [20], and Goyal and Giri [21].

In most of the above-mentioned papers, the demand during stockout period is totally backlogged. In practice, there are customers who are willing to wait and receive their orders at the end of stockout period, while others are not. In the last few years, considerable attention has been paid to inventory models with partial backlogging. The backlogging rate can be modelled taking into account the customers' behavior. The first paper in which customers' impatience functions are proposed seems to be that by Abad [22]. Chang and Dye [23] developed a finite horizon inventory model using Abad's reciprocal backlogging rate. Skouri and Papachristos [24] studied a multiperiod inventory model using the negative exponential backlogging rate proposed by Abad [22]. Teng et al. [25] extended Chang and Dye 's [23] and Skouri and Papachristos' [24] models, assuming as backlogging rate any decreasing function of the waiting time up to the next replenishment. Research on models with partial backlogging continues with Wang [26] and San Jose et al. [27] and [28].

Manna and Chaudhuri [14] noted that ramp type demand pattern is generally followed by new brand of consumer goods coming to the market. But for fashionable products as well as for seasonal products, the steady demand will never be continued indefinitely. Rather it would be followed by decrement with respect to time after a period of time and becomes asymptotic in nature. Thus the demand may be illustrated by three successive time periods that classified time dependent ramp-type function, in which in the first phase the demand increases with time and after that it becomes steady, and towards the end in the final phase it decreases and becomes asymptotic. Chen et al. [29] proposed a search procedure based on Nelder-Mead algorithm to find a solution for the case of inventory systems with shortage allowance and nonlinear demand pattern. Also, Chen et al. [30] proposed a net present value approach for the previous inventory system without shortages. For both models, the demand rate is a revised version of the Beta distribution function and so is a differentiable with respect to time. 
The purpose of the present paper is to study an order level inventory model when the demand is described by a three successive time periods that classified time dependent ramp-type function. Any such function has points, at least one, where differentiation is not possible, and this introduces extra complexity in the analysis of the relevant models. The unsatisfied demand is partially backlogged with time dependent backlogging rate, and units in inventory are subject to deterioration with Weibull-distributed deterioration rate.

The rest of the paper is organized as follows. In the next section the assumptions and notations for the development of the model are provided. The model starting with no shortages is studied in Section 3, and the corresponding one starting with shortages is studied in Section 4. For each model the optimal policy is obtained. Numerical examples highlighting the results obtained are given in Section 5. The paper closes with concluding remarks in Section 6.

\section{Notation and Assumptions}

The following notations and assumptions are used in developing the model:

\section{Notations}

$T$ The constant scheduling period (cycle)

$t_{1}$ The time when the inventory level reaches zero

$S$ The maximum inventory level at each scheduling period (cycle)

$c_{1}$ The inventory holding cost per unit per unit time

$c_{2}$ The shortage cost per unit per unit time

$c_{3}$ The cost incurred from the deterioration of one unit

$c_{4}$ The per unit opportunity cost due to the lost sales

$\mu$ The time point that increasing demand becomes steady

$\gamma$ The time point, after $\mu$, until the demand is steady and then decreases

$I(t)$ The inventory level at time $t \in[0, T]$.

\section{Assumptions}

(1) The ordering quantity brings the inventory level up to the order level $S$. Replenishment rate is infinite.

(2) Shortages are backlogged at a rate $\beta(x)$, which is a nonincreasing function of $x\left(\beta^{\prime}(x) \leq 0\right)$ with $0 \leq \beta(x) \leq 1, \beta(0)=1$, and $x$ is the waiting time up to the next replenishment. Moreover it is assumed that $\beta(x)$ satisfies the relation $\beta(x)+T \beta^{\prime}(x) \geq 0$, where $\beta^{\prime}(x)$ is the derivate of $\beta(x)$. The cases with $\beta(x)=1$ (or 0$)$ correspond to complete backlogging (or complete lost sales) models.

(3) The time to deterioration of the item is distributed as Weibull $(a, b)$; that is, the deterioration rate is $\theta(t)=a b t^{b-1}(a>0, b>0, t>0)$. There is no replacement or repair of deteriorated units during the period $T$. For $b=1, \theta(t)$ becomes constant, which corresponds to exponentially decaying case. 
(4) The demand rate $D(t)$ is a time dependent ramp-type function and is of the following form:

$$
D(t)= \begin{cases}f(t), & 0<t<\mu, \\ f(\mu)=g(\gamma), & \mu \leq t \leq \gamma, \\ g(t), & \gamma<t,\end{cases}
$$

where $f(t)$ is a positive, continuous, and increasing function of $t$, and $g(t)$ is a positive, continuous and decreasing function of $t$.

\section{The Mathematical Formulation of the Model Starting with No Shortages}

The replenishment at the beginning of the cycle brings the inventory level up to $S$. Due to demand and deterioration, the inventory level gradually depletes during the period $\left(0, t_{1}\right)$ and falls to zero at $t=t_{1}$. Thereafter shortages occur during the period $\left(t_{1}, T\right)$, which are partially backlogged. Consequently, the inventory level, $I(t)$, during the time interval $0 \leq t \leq$ $T$, satisfies the following differential equations:

$$
\begin{gathered}
\frac{d I(t)}{d t}+\theta(t) I(t)=-D(t), \quad 0 \leq t \leq t_{1}, I\left(t_{1}\right)=0, \\
\frac{d I(t)}{d t}=-D(t) \beta(T-t), \quad t_{1} \leq t \leq T, I\left(t_{1}\right)=0 .
\end{gathered}
$$

The solutions of these differential equations are affected from the relation between $t_{1}$, $\mu$, and $\gamma$ through the demand rate function. Since the demand has three components in three successive time periods, the following cases: (i) $t_{1}<\mu<\gamma<T$, (ii) $\mu<t_{1}<\gamma<T$, and (iii) $\mu<\gamma<t_{1}<T$ must be considered to determine the total cost and then the optimal replenishment policy.

Case $1\left(t_{1}<\mu<\gamma<T\right)$. In this case, (3.1) becomes

$$
\frac{d I(t)}{d t}+a b t^{b-1} I(t)=-f(t), \quad 0 \leq t \leq t_{1}, I\left(t_{1}\right)=0 .
$$

Equation (3.2) leads to the following three:

$$
\begin{gathered}
\frac{d I(t)}{d t}=-f(t) \beta(T-t), \quad t_{1} \leq t \leq \mu, I\left(t_{1}\right)=0, \\
\frac{d I(t)}{d t}=-f(\mu) \beta(T-t), \quad \mu \leq t \leq \gamma, I\left(\mu_{-}\right)=I\left(\mu_{+}\right), \\
\frac{d I(t)}{d t}=-g(t) \beta(T-t), \quad \gamma \leq t \leq T .
\end{gathered}
$$


The solutions of (3.3), (3.4), (3.5), and (3.6), are, respectively,

$$
\begin{gathered}
I(t)=e^{-a t^{b}} \int_{t}^{t_{1}} f(x) e^{a x^{b}} d x, \quad 0 \leq t \leq t_{1}, \\
I(t)=-\int_{t_{1}}^{t} f(x) \beta(T-x) d x, \quad t_{1} \leq t \leq \mu, \\
I(t)=-f(\mu) \int_{\mu}^{t} \beta(T-x) d x-\int_{t_{1}}^{\mu} f(x) \beta(T-x) d x, \quad \mu \leq t \leq \gamma, \\
I(t)=-\int_{\gamma}^{t} g(x) \beta(T-x) d x-\int_{t_{1}}^{\mu} f(x) \beta(T-x) d x-f(\mu) \int_{\mu}^{\gamma} \beta(T-x) d x, \quad \gamma \leq t \leq T .
\end{gathered}
$$

The total amount of deteriorated items during $\left[0, t_{1}\right]$ is

$$
D=\int_{0}^{t_{1}} f(t) e^{a t^{b}} d t-\int_{0}^{t_{1}} f(t) d t
$$

The cumulative inventory carried in the interval $\left[0, t_{1}\right]$ is found from (3.7) and is

$$
I_{1}=\int_{0}^{t_{1}} I(t) d t=\int_{0}^{t_{1}} e^{-a t^{b}}\left[\int_{t}^{t_{1}} f(x) e^{a x^{b}} d x\right] d t
$$

Due to (3.8), (3.9), and (3.10), the time-weighted backorders during the interval $\left[t_{1}, T\right]$ are

$$
\begin{aligned}
I_{2}= & \int_{t_{1}}^{T}[-I(t)] d t \\
= & \int_{t_{1}}^{\mu}[-I(t)] d t+\int_{\mu}^{\gamma}[-I(t)] d t+\int_{\gamma}^{T}[-I(t)] d t \\
= & \int_{t_{1}}^{\mu}(\mu-t) f(t) \beta(T-t) d t+f(\mu) \int_{\mu}^{\gamma}\left[\int_{\mu}^{t} \beta(T-x) d x\right] d t+\int_{\mu}^{\gamma}\left[\int_{t_{1}}^{\mu} f(x) \beta(T-x) d x\right] d t \\
& +\int_{\gamma}^{T}\left[\int_{\gamma}^{t} g(x) \beta(T-x) d x\right] d t+\int_{\gamma}^{T}\left[\int_{t_{1}}^{\mu} f(x) \beta(T-x) d x\right] d t+f(\mu) \int_{\gamma}^{T}\left[\int_{\mu}^{\gamma} \beta(T-x) d x\right] d t .
\end{aligned}
$$

The amount of lost sales during $\left[t_{1}, T\right]$ is

$$
L=\int_{t_{1}}^{\mu}(1-\beta(T-t)) f(t) d t+f(\mu) \int_{\mu}^{\gamma}(1-\beta(T-t)) d t+\int_{\gamma}^{T}(1-\beta(T-t)) g(t) d t .
$$


The total cost in the time interval $[0, T]$ is the sum of holding, shortage, deterioration, and opportunity costs and is given by

$$
\begin{aligned}
T C_{1}\left(t_{1}\right)= & c_{1} I_{1}+c_{2} I_{2}+c_{3} D+c_{4} L \\
= & c_{1}\left\{\int_{0}^{t_{1}} e^{-a t^{b}}\left[\int_{t}^{t_{1}} f(x) e^{a x^{b}} d x\right] d t\right\}+c_{3}\left\{\int_{0}^{t_{1}} f(t) e^{a t^{b}} d t-\int_{0}^{t_{1}} f(t) d t\right\} \\
& +c_{2}\left\{\int_{t_{1}}^{\mu}(\mu-t) f(t) \beta(T-t) d t+f(\mu) \int_{\mu}^{\gamma}\left[\int_{\mu}^{t} \beta(T-x) d x\right] d t\right. \\
& +\int_{\mu}^{\gamma}\left[\int_{t_{1}}^{\mu} f(x) \beta(T-x) d x\right] d t+\int_{\gamma}^{T}\left[\int_{\gamma}^{t} g(x) \beta(T-x) d x\right] d t \\
& \left.+\int_{\gamma}^{T}\left[\int_{t_{1}}^{\mu} f(x) \beta(T-x) d x\right] d t+f(\mu) \int_{\gamma}^{T}\left[\int_{\mu}^{\gamma} \beta(T-x) d x\right] d t\right\} \\
& +c_{4}\left\{\int_{t_{1}}^{\mu}(1-\beta(T-t)) f(t) d t+f(\mu) \int_{\mu}^{\gamma}(1-\beta(T-t)) d t+\int_{\gamma}^{T}(1-\beta(T-t)) g(t) d t\right\} .
\end{aligned}
$$

Case $2\left(\mu<t_{1}<\gamma<T\right)$. In this case, (3.1) reduces to the following two:

$$
\begin{aligned}
& \frac{d I(t)}{d t}+a b t^{b-1} I(t)=-f(t), \quad 0 \leq t \leq \mu, I\left(\mu^{-}\right)=I\left(\mu^{+}\right), \\
& \frac{d I(t)}{d t}+a b t^{b-1} I(t)=-f(\mu), \quad \mu \leq t \leq t_{1}, \quad I\left(t_{1}\right)=0 .
\end{aligned}
$$

Equation (3.2) leads to the following two:

$$
\begin{array}{ll}
\frac{d I(t)}{d t}=-f(\mu) \beta(T-t), & t_{1} \leq t \leq \gamma, I\left(t_{1}\right)=0, \\
\frac{d I(t)}{d t}=-g(t) \beta(T-t), & \gamma \leq t \leq T, I\left(\gamma^{-}\right)=I\left(\gamma^{+}\right) .
\end{array}
$$

Their solutions are, respectively,

$$
\begin{aligned}
& I(t)=e^{-a t^{b}}\left[\int_{t}^{\mu} f(x) e^{a x^{b}} d x+f(\mu) \int_{\mu}^{t_{1}} e^{a x^{b}} d x\right], \quad 0 \leq t \leq \mu, \\
& I(t)=e^{-a t^{b}} f(\mu) \int_{t}^{t_{1}} e^{a x^{b}} d x, \quad \mu \leq t \leq t_{1}, \\
& I(t)=-f(\mu) \int_{t_{1}}^{t} \beta(T-x) d x, \quad t_{1} \leq t \leq \gamma, \\
& I(t)=-\int_{\gamma}^{t} g(x) \beta(T-x) d x-f(\mu) \int_{t_{1}}^{\gamma} \beta(T-x) d x, \quad \gamma \leq t \leq T .
\end{aligned}
$$


The total amount of deteriorated items during $\left[0, t_{1}\right]$ is

$$
D=I(0)-\int_{0}^{t_{1}} D(t) d t=\int_{0}^{\mu} f(t) e^{a t^{b}} d t+f(\mu) \int_{\mu}^{t_{1}} e^{a t^{b}} d t-\int_{0}^{\mu} f(t) d t-f(\mu)\left(t_{1}-\mu\right)
$$

The total inventory carried during the interval $\left[0, t_{1}\right]$ is

$$
\begin{aligned}
I_{1} & =\int_{0}^{t_{1}} I(t) d t=\int_{0}^{\mu} I(t) d t+\int_{\mu}^{t_{1}} I(t) d t \\
& =\int_{0}^{\mu} e^{-a t^{b}}\left[\int_{t}^{\mu} f(x) e^{a x^{b}} d x+f(\mu) \int_{\mu}^{t_{1}} e^{a x^{b}} d x\right] d t+f(\mu) \int_{\mu}^{t_{1}} e^{-a t^{b}}\left[\int_{t}^{t_{1}} e^{a x^{b}} d x\right] d t .
\end{aligned}
$$

The time-weighted backorders during the interval $\left[t_{1}, T\right]$ are

$$
\begin{aligned}
I_{2} & =\int_{t_{1}}^{T}[-I(t)] d t=\int_{t_{1}}^{\gamma}[-I(t)] d t+\int_{\gamma}^{T}[-I(t)] d t \\
& =\int_{t_{1}}^{\gamma} f(\mu) \int_{t_{1}}^{t} \beta(T-x) d x d t+\int_{\gamma}^{T} \int_{\gamma}^{t} g(x) \beta(T-x) d x d t+f(\mu) \int_{\gamma}^{T} \int_{t_{1}}^{\gamma} \beta(T-x) d x d t .
\end{aligned}
$$

The lost sales in the interval $\left[t_{1}, T\right]$ are

$$
L=f(\mu) \int_{t_{1}}^{\gamma}[1-\beta(T-t)] d t+\int_{\gamma}^{T}[1-\beta(T-t)] g(t) d t .
$$

The inventory cost for this case is

$$
\begin{aligned}
T C_{2}\left(t_{1}\right)= & c_{1} I_{1}+c_{2} I_{2}+c_{3} D+c_{4} L \\
= & c_{1}\left\{\int_{0}^{\mu} e^{-a t^{b}}\left[\int_{t}^{\mu} f(x) e^{a x^{b}} d x+f(\mu) \int_{\mu}^{t_{1}} e^{a x^{b}} d x\right] d t+f(\mu) \int_{\mu}^{t_{1}} e^{-a t^{b}}\left[\int_{t}^{t_{1}} e^{a x^{b}} d x\right] d t\right\} \\
& +c_{2}\left\{\int_{t_{1}}^{\gamma} f(\mu) \int_{t_{1}}^{t} \beta(T-x) d x d t+\int_{\gamma}^{T} \int_{\gamma}^{t} g(x) \beta(T-x) d x d t\right. \\
& \left.+f(\mu) \int_{\gamma}^{T} \int_{t_{1}}^{\gamma} \beta(T-x) d x d t\right\} \\
& +c_{3}\left\{\int_{0}^{\mu} f(t) e^{a t^{b}} d t+f(\mu) \int_{\mu}^{t_{1}} e^{a t^{b}} d t-\int_{0}^{\mu} f(t) d t-f(\mu)\left(t_{1}-\mu\right)\right\} \\
& +c_{4}\left\{f(\mu) \int_{t_{1}}^{\gamma}[1-\beta(T-t)] d t+\int_{\gamma}^{T}[1-\beta(T-t)] g(t) d t\right\} .
\end{aligned}
$$


Case $3\left(\mu<\gamma<t_{1}<T\right)$. In this case, (3.1) reduces to the following three:

$$
\begin{array}{ll}
\frac{d I(t)}{d t}+a b t^{b-1} I(t)=-f(t), & 0 \leq t \leq \mu, I\left(\mu^{-}\right)=I\left(\mu^{+}\right), \\
\frac{d I(t)}{d t}+a b t^{b-1} I(t)=-f(\mu), & \mu \leq t \leq \gamma, I\left(\gamma^{-}\right)=I\left(\gamma^{+}\right), \\
\frac{d I(t)}{d t}+a b t^{b-1} I(t)=-g(t), & \gamma \leq t \leq t_{1}, I\left(t_{1}\right)=0 .
\end{array}
$$

Equation (3.2) leads to the following:

$$
\frac{d I(t)}{d t}=-g(t) \beta(T-t), \quad t_{1} \leq t \leq T, I\left(t_{1}\right)=0 .
$$

Their solutions are, respectively,

$$
\begin{aligned}
& I(t)=e^{-a t^{b}}\left[\int_{t}^{\mu} f(x) e^{a x^{b}} d x+f(\mu) \int_{\mu}^{\gamma} e^{a x^{b}} d x+\int_{\gamma}^{t_{1}} g(x) e^{a x^{b}} d x\right], \quad 0 \leq t \leq \mu, \\
& I(t)=e^{-a t^{b}}\left[f(\mu) \int_{t}^{\gamma} e^{a x^{b}} d x+\int_{\gamma}^{t_{1}} g(x) e^{a x^{b}} d x\right], \quad \mu \leq t \leq \gamma, \\
& I(t)=e^{-a t^{b}} \int_{t}^{t_{1}} g(x) e^{a x^{b}} d x, \quad \gamma \leq t \leq t_{1}, \\
& I(t)=-\int_{t_{1}}^{t} g(x) \beta(T-x) d x, \quad t_{1} \leq t \leq T .
\end{aligned}
$$

The total amount of deteriorated items during $\left[0, t_{1}\right]$ is

$$
\begin{aligned}
D= & I(0)-\int_{0}^{t_{1}} D(t) d t \\
= & \int_{0}^{\mu} f(x) e^{a x^{b}} d x+f(\mu) \int_{\mu}^{\gamma} e^{a x^{b}} d x+\int_{\gamma}^{t_{1}} g(x) e^{a x^{b}} d x \\
& -\int_{0}^{\mu} f(t) d t-f(\mu)(\gamma-\mu)-\int_{\gamma}^{t_{1}} g(t) d t .
\end{aligned}
$$


The total inventory carried during the interval $\left[0, t_{1}\right]$, using (3.26), (3.27), and (3.28) is

$$
\begin{aligned}
I_{1}= & \int_{0}^{t_{1}} I(t) d t=\int_{0}^{\mu} I(t) d t+\int_{\mu}^{\gamma} I(t) d t+\int_{\gamma}^{t_{1}} I(t) d t \\
= & \int_{0}^{\mu} e^{-a t^{b}}\left[\int_{t}^{\mu} f(x) e^{a x^{b}} d x+f(\mu) \int_{\mu}^{\gamma} e^{a x^{b}} d x+\int_{\gamma}^{t_{1}} g(x) e^{a x^{b}} d x\right] d t \\
& +\int_{\mu}^{\gamma} e^{-a t^{b}}\left[f(\mu) \int_{t}^{\gamma} e^{a x^{b}} d x+\int_{\gamma}^{t_{1}} g(x) e^{a x^{b}} d x\right] d t+\int_{\gamma}^{t_{1}} e^{-a t^{b}}\left[\int_{t}^{t_{1}} g(x) e^{a x^{b}} d x\right] d t .
\end{aligned}
$$

The time-weighted backorders during the interval $\left[t_{1}, T\right]$ are

$$
I_{2}=\int_{t_{1}}^{T}[-I(t)] d t=\int_{t_{1}}^{T} \int_{t_{1}}^{t} g(x) \beta(T-x) d x
$$

The lost sales in the interval $\left[t_{1}, T\right]$ are

$$
L=\int_{t_{1}}^{T}[1-\beta(T-t)] g(t) d t
$$

The inventory cost for this case is

$$
\begin{aligned}
& T C_{3}\left(t_{1}\right)=c_{1} I_{1}+c_{2} I_{2}+c_{3} D+c_{4} L \\
& =c_{1}\left\{\int_{0}^{\mu} e^{-a t^{b}}\left[\int_{t}^{\mu} f(x) e^{a x^{b}} d x+f(\mu) \int_{\mu}^{\gamma} e^{a x^{b}} d x+\int_{\gamma}^{t_{1}} g(x) e^{a x^{b}} d x\right] d t\right. \\
& \left.\quad+\int_{\mu}^{\gamma} e^{-a t^{b}}\left[f(\mu) \int_{t}^{\gamma} e^{a x^{b}} d x+\int_{\gamma}^{t_{1}} g(x) e^{a x^{b}} d x\right] d t+\int_{\gamma}^{t_{1}} e^{-a t^{b}}\left[\int_{t}^{t_{1}} g(x) e^{a x^{b}} d x\right] d t\right\} \\
& +c_{2}\left\{\int_{t_{1}}^{T} \int_{t_{1}}^{t} g(x) \beta(T-t) d x\right\}+c_{4}\left\{\int_{t_{1}}^{T}[1-\beta(T-t)] g(t) d t\right\} \\
& +c_{3}\left\{\int_{0}^{\mu} f(x) e^{a x^{b}} d x+f(\mu) \int_{\mu}^{\gamma} e^{a x^{b}} d x+\int_{\gamma}^{t_{1}} g(x) e^{a x^{b}} d x\right. \\
& \left.\quad-\int_{0}^{\mu} f(t) d t-f(\mu)(\gamma-\mu)-\int_{\gamma}^{t_{1}} g(t) d t\right\} .
\end{aligned}
$$

Finally the total cost function of the system over $[0, T]$ takes the following form:

$$
T C\left(t_{1}\right)= \begin{cases}T C_{1}\left(t_{1}\right), & \text { if } t_{1} \leq \mu, \\ T C_{2}\left(t_{1}\right), & \text { if } \mu<t_{1}<\gamma, \\ T C_{3}\left(t_{1}\right), & \text { if } \gamma \leq t_{1} .\end{cases}
$$


It is easy to check that this function is continuous at $\mu$ and $\gamma$. The problem now is the minimization of this, three branches, function $T C\left(t_{1}\right)$. This requires, separately, studying each of these branches and then combining the results to state the algorithm giving the optimal policy.

\subsection{The Optimal Replenishment Policy}

In this subsection we present the results, which ensure the existence of a unique optimal value for $t_{1}$, say $t_{1}^{*}$, which minimizes the total cost function. Although the optimality procedure requires the constrained optimization of the functions $T C_{1}\left(t_{1}\right), T C_{2}\left(t_{1}\right)$, and $T C_{3}\left(t_{1}\right)$, we will, firstly, search for their unconstrained minimum. The first- and second-order derivatives of $T C_{1}\left(t_{1}\right), T C_{2}\left(t_{1}\right)$, and $T C_{3}\left(t_{1}\right)$ are, respectively,

$$
\begin{aligned}
& \frac{d T C_{1}\left(t_{1}\right)}{d t_{1}}=f\left(t_{1}\right) h\left(t_{1}\right), \\
& \frac{d^{2} T C_{1}\left(t_{1}\right)}{d t_{1}^{2}}=\frac{d f\left(t_{1}\right)}{d t_{1}} h\left(t_{1}\right)+f\left(t_{1}\right) \frac{d h\left(t_{1}\right)}{d t_{1}}, \\
& \frac{d T C_{2}\left(t_{1}\right)}{d t_{1}}=f(\mu) h\left(t_{1}\right), \\
& \frac{d^{2} T C_{2}\left(t_{1}\right)}{d t_{1}^{2}}=f(\mu) \frac{d h\left(t_{1}\right)}{d t_{1}}, \\
& \frac{d T C_{3}\left(t_{1}\right)}{d t_{1}}=g\left(t_{1}\right) h\left(t_{1}\right), \\
& \frac{d^{2} T C_{3}\left(t_{1}\right)}{d t_{1}^{2}}=\frac{d g\left(t_{1}\right)}{d t_{1}} h\left(t_{1}\right)+g\left(t_{1}\right) \frac{d h\left(t_{1}\right)}{d t_{1}},
\end{aligned}
$$

where

$$
h\left(t_{1}\right)=c_{1} e^{a t_{1} b} \int_{0}^{t_{1}} e^{-a t^{b}} d t+c_{3}\left(e^{a t_{1} b^{b}}-1\right)-c_{2}\left(T-t_{1}\right) \beta\left(T-t_{1}\right)-c_{4}\left(1-\beta\left(T-t_{1}\right)\right) .
$$

Equation (3.37) is the same as (16) of the paper of Skouri et al. [16]. So, following the methodology proposed by Skouri et al. [16], the algorithm, which gives the optimal replenishment policy, is as follows.

Step 1. Compute $t_{1}^{*}$ from $h\left(t_{1}\right)=0$.

Step 2. If $t_{1}^{*} \leq \mu$, then the optimal order quantity is given by

$$
Q^{*}=\int_{0}^{t_{1}^{*}} f(t) e^{a t^{b}} d t+\int_{t_{1}^{*}}^{\mu} \beta(T-t) f(t) d t+f(\mu) \int_{\mu}^{\gamma} \beta(T-t) d t+\int_{\gamma}^{T} \beta(T-t) g(t) d t
$$

and the total cost is given by $T C_{1}\left(t_{1}^{*}\right)$. 
If $\mu<t_{1}^{*}<\gamma$, then the optimal order quantity is given by

$$
Q^{*}=\int_{0}^{\mu} f(t) e^{a t^{b}} d t+f(\mu) \int_{\mu}^{t_{1}^{*}} e^{a t^{b}} d t+f(\mu) \int_{t_{1}^{*}}^{\gamma} \beta(T-t) d t+\int_{\gamma}^{T} g(t) \beta(T-t) d t
$$

and the total cost is given by $T C_{2}\left(t_{1}^{*}\right)$.

If $\gamma<t_{1}^{*}<T$, then the optimal order quantity is given by

$$
Q^{*}=\int_{0}^{\mu} f(t) e^{a t^{b}} d t+f(\mu) \int_{\mu}^{\gamma} e^{a t^{b}} d t+\int_{\gamma}^{t_{1}^{*}} g(t) e^{a t^{b}} d t+\int_{t_{1}^{*}}^{T} g(t) \beta(T-t) d t
$$

and the total cost is given by $T C_{3}\left(t_{1}^{*}\right)$.

Remark 3.1. The previous analysis shows that $t_{1}^{*}$ is independent from the demand rate $D(t)$. This very interesting result agrees with the classical result, in many order level inventory systems, that the point $t_{1}^{*}$ is independent from the demand rate (Naddor [31, page 67]).

\subsection{The Special Case $\beta(x)=1$ and $a=0$}

If we are considering the case that there is no deterioration of the product $(a=0)$ and unsatisfied demand is complete backlogged $(\beta(x)=1)$, then the total cost function of the model starting with no shortages over $[0, T]$ takes the following form:

$$
T C\left(t_{1}\right)=\left\{\begin{array}{cc}
c_{1} \int_{0}^{t_{1}} \int_{t}^{t_{1}} f(x) d x d t+c_{2} \int_{t_{1}}^{\mu} \int_{t_{1}}^{t} f(x) d x d t+c_{2} f(\mu) \int_{\mu}^{\gamma}(t-\mu) d t+c_{2} \int_{\mu}^{\gamma} \int_{t_{1}}^{\mu} f(x) d x d t \\
+c_{2} \int_{\gamma}^{T} \int_{\gamma}^{t} g(x) d x d t d t+c_{2} f(\mu)(\gamma-\mu)(T-\gamma)+c_{2} \int_{\gamma}^{T} \int_{t_{1}}^{\mu} f(x) d x d t, \\
c_{1} \int_{0}^{\mu} \int_{t}^{\mu} f(x) d x d t+c_{1} \int_{0}^{\mu} \int_{\mu}^{t_{1}} f(\mu) d x d t+c_{1} \int_{\mu}^{t_{1}} \int_{t}^{t_{1}} f(\mu) d x d t+c_{2} \int_{t_{1}}^{\gamma} \int_{t_{1}}^{t} f(\mu) d x d t \\
+c_{2} \int_{\gamma}^{T} \int_{\gamma}^{t} g(x) d x d t+c_{2} \int_{\gamma}^{T} \int_{t_{1}}^{\gamma} f(\mu) d x d t, \quad \mu<t_{1}<\gamma<T, \\
c_{1} \int_{0}^{\mu} \int_{t}^{\mu} f(x) d x d t+c_{1} \int_{0}^{\mu} \int_{\mu}^{\gamma} f(\mu) d x d t+c_{1} \int_{0}^{\mu} \int_{\gamma}^{t_{1}} g(x) d x d t+c_{1} \int_{\mu}^{\gamma} \int_{t}^{\gamma} f(\mu) d x d t \\
+c_{1} \int_{\mu}^{\gamma} \int_{\gamma}^{t_{1}} g(x) d x d t+c_{1} \int_{\gamma}^{t_{1}} \int_{t}^{t_{1}} g(x) d x d t+c_{2} \int_{t_{1}}^{T} \int_{t_{1}}^{t} g(x) d x d t, \quad \mu<\gamma<t_{1}<T .
\end{array}\right.
$$


Following the previous procedure for the optimal replenishment policy, the optimal value of $t_{1}$, say $t_{1}^{*}$, is given by the very simple and known, in classical order level inventory system (Naddor [31]), equation:

$$
t_{1}^{*}=\frac{c_{2} T}{c_{1}+c_{2}}
$$

\section{The Mathematical Formulation of the Model Starting with Shortages}

In this section the inventory model starting with shortages is studied. The cycle now starts with shortages, which occur during the period $\left(0, t_{1}\right)$, and are partially backlogged. At time $t_{1}$ a replenishment brings the inventory level up to $S$. Demand and deterioration of the items deplete the inventory level during the period $\left(t_{1}, T\right)$ until this falls to zero at $t=T$. Again the three cases $t_{1}<\mu<\gamma<T, \mu<t_{1}<\gamma<T$, and $\mu<\gamma<t_{1}<T$ must be examined.

Case $4\left(t_{1}<\mu<\gamma<T\right)$. The inventory level, $I(t), 0 \leq t \leq T$ satisfies the following differential equations:

$$
\begin{aligned}
& \frac{d I(t)}{d t}=-f(t) \beta\left(t_{1}-t\right), \quad 0 \leq t \leq t_{1}, I(0)=0, \\
& \frac{d I(t)}{d t}+a b t^{b-1} I(t)=-f(t), \quad t_{1} \leq t \leq \mu, I\left(\mu_{-}\right)=I\left(\mu_{+}\right), \\
& \frac{d I(t)}{d t}+a b t^{b-1} I(t)=-f(\mu), \quad \mu \leq t \leq \gamma, I\left(\gamma_{-}\right)=I\left(\gamma_{+}\right), \\
& \frac{d I(t)}{d t}+a b t^{b-1} I(t)=-g(t), \quad \gamma \leq t \leq T, I(T)=0 .
\end{aligned}
$$

The solutions of (4.1), are, respectively,

$$
\begin{gathered}
I(t)=-\int_{0}^{t} f(x) \beta\left(t_{1}-x\right) d x, \quad 0 \leq t \leq t_{1}, \\
I(t)=e^{-\alpha t^{b}}\left[\int_{t}^{\mu} e^{\alpha x^{b}} f(x) d x+f(\mu) \int_{\mu}^{\gamma} e^{\alpha x^{b}} d x+\int_{\gamma}^{T} e^{\alpha x^{b}} g(x) d x\right], \quad t_{1} \leq t \leq \mu, \\
I(t)=e^{-\alpha t^{b}}\left[f(\mu) \int_{t}^{\gamma} e^{\alpha x^{b}} d x+\int_{\gamma}^{T} e^{\alpha x^{b}} g(x) d x\right], \quad \mu \leq t \leq \gamma, \\
I(t)=e^{-a t^{b}} \int_{t}^{T} e^{a x^{b}} g(x) d x, \quad \gamma \leq t \leq T .
\end{gathered}
$$

The total amount of deteriorated units during $\left[t_{1}, T\right]$ is

$$
\begin{aligned}
D= & e^{-a t_{1}^{b}}\left[\int_{t_{1}}^{\mu} f(x) e^{a x^{b}} d x+f(\mu) \int_{\mu}^{\gamma} e^{\alpha x^{b}} d x+\int_{\gamma}^{T} e^{\alpha x^{b}} g(x) d x\right] \\
& -\int_{t_{1}}^{\mu} f(x) d x-(\gamma-\mu) f(\mu)-\int_{\gamma}^{T} g(x) d x .
\end{aligned}
$$


The total inventory carried during the interval $\left[t_{1}, T\right]$ is found using (4.3), (4.4), and (4.5) and is

$$
\begin{aligned}
I_{1}= & \int_{t_{1}}^{\mu} e^{-a t^{b}}\left[\int_{t}^{\mu} e^{\alpha x^{b}} f(x) d x+f(\mu) \int_{\mu}^{\gamma} e^{\alpha x^{b}} d x+\int_{\gamma}^{T} e^{\alpha x^{b}} g(x) d x\right] d t \\
& +\int_{\mu}^{\gamma} e^{-\alpha t^{b}}\left[f(\mu) \int_{t}^{\gamma} e^{\alpha x^{b}} d x+\int_{\gamma}^{T} e^{\alpha x^{b}} g(x) d x\right] d t+\int_{\gamma}^{T} e^{-a t^{b}}\left[\int_{t}^{T} e^{a x^{b}} g(x) d x\right] d t .
\end{aligned}
$$

Due to (4.2) the time-weighted backorders during the time interval $\left[0, t_{1}\right]$ are

$$
I_{2}=\int_{0}^{t_{1}} \int_{0}^{t} f(x) \beta\left(t_{1}-x\right) d x d t
$$

The amount of lost sales during $\left[0, t_{1}\right]$ is

$$
L=\int_{0}^{t_{1}}\left[1-\beta\left(t_{1}-t\right)\right] f(t) d t
$$

The inventory cost during the time interval $[0, T]$ is the sum of holding, shortage, deterioration, and opportunity costs and is given by

$$
\begin{aligned}
T C_{1}\left(t_{1}\right)= & c_{1} I_{1}+c_{2} I_{2}+c_{3} D+c_{4} L \\
= & c_{1}\left(\int_{t_{1}}^{\mu} e^{-a t^{b}}\left[\int_{t}^{\mu} e^{\alpha x^{b}} f(x) d x+f(\mu) \int_{\mu}^{\gamma} e^{\alpha x^{b}} d x+\int_{\gamma}^{T} e^{\alpha x^{b}} g(x) d x\right] d t\right) \\
& +c_{1}\left(\int_{\mu}^{\gamma} e^{-\alpha t^{b}}\left[f(\mu) \int_{t}^{\gamma} e^{\alpha x^{b}} d x+\int_{\gamma}^{T} e^{\alpha x^{b}} g(x) d x\right] d t+\int_{\gamma}^{T} e^{-a t^{b}}\left[\int_{t}^{T} e^{a x^{b}} g(x) d x\right] d t\right) \\
& +c_{2} \int_{0}^{t_{1}} \int_{0}^{t} f(x) \beta\left(t_{1}-x\right) d x d t+c_{4} \int_{0}^{t_{1}}\left[1-\beta\left(t_{1}-t\right)\right] f(t) d t \\
& +c_{3}\left[e^{-a t_{1}^{b}}\left[\int_{t_{1}}^{\mu} f(x) e^{a x^{b}} d x+f(\mu) \int_{\mu}^{\gamma} e^{\alpha x^{b}} d x+\int_{\gamma}^{T} e^{\alpha x^{b}} g(x) d x\right]\right. \\
& \left.\quad \int_{t_{1}}^{\mu} f(x) d x-(\gamma-\mu) f(\mu)-\int_{\gamma}^{T} g(x) d x\right] .
\end{aligned}
$$


Case $5\left(\mu<t_{1}<\gamma<T\right)$. The inventory level, $I(t), 0 \leq t \leq T$ satisfies the following differential equations:

$$
\begin{gathered}
\frac{d I(t)}{d t}=-f(t) \beta\left(t_{1}-t\right), \quad 0 \leq t \leq \mu, I(0)=0, \\
\frac{d I(t)}{d t}=-f(\mu) \beta\left(t_{1}-t\right), \quad \mu \leq t \leq t_{1}, I\left(\mu^{-}\right)=I\left(\mu^{+}\right), \\
\frac{d I(t)}{d t}+a b t^{b-1} I(t)=-f(\mu), \quad t_{1} \leq t \leq \gamma, \quad I\left(\gamma_{-}\right)=I\left(\gamma_{+}\right), \\
\frac{d I(t)}{d t}+a b t^{b-1} I(t)=-g(t), \quad \gamma \leq t \leq T, I(T)=0 .
\end{gathered}
$$

The solutions of (4.11), are, respectively,

$$
\begin{aligned}
& I(t)=-\int_{0}^{t} f(x) \beta\left(t_{1}-x\right) d x, \quad 0 \leq t \leq \mu, \\
& I(t)=-\int_{0}^{\mu} f(x) \beta\left(t_{1}-x\right) d x-f(\mu) \int_{\mu}^{t} \beta\left(t_{1}-x\right) d x, \quad \mu \leq t \leq t_{1}, \\
& I(t)=e^{-\alpha t^{b}}\left[f(\mu) \int_{t}^{\gamma} e^{\alpha x^{b}} d x+\int_{\gamma}^{T} e^{\alpha x^{b}} g(x) d x\right], \quad t_{1} \leq t \leq \gamma, \\
& I(t)=e^{-a t^{b}} \int_{t}^{T} e^{a x^{b}} g(x) d x, \quad \gamma \leq t \leq T .
\end{aligned}
$$

The total cost of this case is obtained with a similar way of the previous cases and is,

$$
\begin{aligned}
T C_{2}\left(t_{1}\right)=c_{1}\left[\int_{t_{1}}^{\gamma} e^{-\alpha t^{b}}\left(f(\mu) \int_{t}^{\gamma} e^{\alpha x^{b}} d x+\int_{\gamma}^{T} e^{\alpha x^{b}} g(x) d x\right) d t\right. \\
\left.+\int_{\gamma}^{T}\left(e^{-a t^{b}} \int_{t}^{T} e^{a x^{b}} g(x) d x\right) d t\right] \\
+c_{3}\left[e^{-a t_{1}^{b}}\left(\int_{t_{1}}^{\gamma} f(\mu) e^{a x^{b}} d x+\int_{\gamma}^{T} e^{a x^{b}} g(x) d x\right)-f(\mu)\left(\gamma-t_{1}\right)-\int_{\gamma}^{T} g(x) d x\right] \\
+c_{2}\left[\int_{0}^{\mu}\left[\int_{0}^{t} f(x) \beta\left(t_{1}-x\right) d x\right] d t\right. \\
\left.+\int_{\mu}^{t_{1}}\left[\int_{0}^{\mu} f(x) \beta\left(t_{1}-x\right) d x+f(\mu) \int_{\mu}^{t} \beta\left(t_{1}-x\right) d x\right] d t\right] \\
+c_{4}\left[\int_{0}^{\mu}\left[1-\beta\left(t_{1}-t\right)\right] f(t) d t+f(\mu) \int_{\mu}^{t_{1}}\left[1-\beta\left(t_{1}-t\right)\right] d t\right] .
\end{aligned}
$$


Case $6\left(\mu<\gamma<t_{1}<T\right)$. The inventory level, $I(t), 0 \leq t \leq T$ for this case satisfies the following differential equations:

$$
\begin{gathered}
\frac{d I(t)}{d t}=-f(t) \beta\left(t_{1}-t\right), \quad 0 \leq t \leq \mu, I(0)=0, \\
\frac{d I(t)}{d t}=-f(\mu) \beta\left(t_{1}-t\right), \quad \mu \leq t \leq \gamma, I\left(\mu^{-}\right)=I\left(\mu^{+}\right), \\
\frac{d I(t)}{d t}=-g(t) \beta\left(t_{1}-t\right), \quad \gamma \leq t \leq t_{1}, I\left(\gamma_{-}\right)=I\left(\gamma_{+}\right), \\
\frac{d I(t)}{d t}+a b t^{b-1} I(t)=-g(t), \quad t_{1} \leq t \leq T, I(T)=0 .
\end{gathered}
$$

The solutions of (4.14), (4.15), (4.16), and (4.17), are, respectively,

$$
\begin{aligned}
& I(t)=-\int_{0}^{t} f(x) \beta\left(t_{1}-x\right) d x, \quad 0 \leq t \leq \mu, \\
& I(t)=-\int_{0}^{\mu} f(x) \beta\left(t_{1}-x\right) d x-f(\mu) \int_{\mu}^{t} \beta\left(t_{1}-x\right) d x, \quad \mu \leq t \leq r, \\
& I(t)=-\int_{\gamma}^{t} g(x) \beta\left(t_{1}-x\right) d x-\int_{0}^{\mu} f(x) \beta\left(t_{1}-x\right) d x-f(\mu) \int_{\mu}^{\gamma} \beta\left(t_{1}-x\right) d x, \quad \gamma \leq t \leq t_{1}, \\
& I(t)=e^{-a t^{b}} \int_{t}^{T} e^{a x^{b}} g(x) d x, \quad t_{1} \leq t \leq T .
\end{aligned}
$$

The total cost of this case is obtained with a similar way of the previous cases and is,

$$
\begin{aligned}
T C_{3}\left(t_{1}\right)= & c_{1}\left[\int_{t_{1}}^{T} e^{-\alpha t^{b}} \int_{t}^{T} e^{a x^{b}} g(x) d x d t\right]+c_{3}\left[e^{-a t_{1}^{b}} \int_{t_{1}}^{T} e^{a x^{b}} g(x) d x-\int_{t_{1}}^{T} g(x) d x\right] \\
& +c_{2}\left[\int_{0}^{\mu}\left[\int_{0}^{t} f(x) \beta\left(t_{1}-x\right) d x\right] d t+\int_{\mu}^{\gamma}\left[\int_{0}^{\mu} f(x) \beta\left(t_{1}-x\right) d x+f(\mu) \int_{\mu}^{t} \beta\left(t_{1}-x\right) d x\right] d t\right] \\
& +c_{2}\left[\int_{\gamma}^{t_{1}}\left(\int_{\gamma}^{t} g(x) \beta\left(t_{1}-x\right) d x+\int_{0}^{\mu} f(x) \beta\left(t_{1}-x\right) d x+f(\mu) \int_{\mu}^{\gamma} \beta\left(t_{1}-x\right) d x\right) d t\right] \\
& +c_{4}\left[\int_{0}^{\mu}\left[1-\beta\left(t_{1}-t\right)\right] f(t) d t+f(\mu) \int_{\mu}^{\gamma}\left[1-\beta\left(t_{1}-t\right)\right] d t+\int_{\gamma}^{t_{1}}\left[1-\beta\left(t_{1}-t\right)\right] g(t) d t\right] .
\end{aligned}
$$


Finally the total cost function of the system over $[0, T]$ takes the following form:

$$
T C\left(t_{1}\right)= \begin{cases}T C_{1}\left(t_{1}\right), & \text { if } t_{1} \leq \mu, \\ T C_{2}\left(t_{1}\right), & \text { if } \mu<t_{1}<\gamma, \\ T C_{3}\left(t_{1}\right), & \text { if } \gamma \leq t_{1} .\end{cases}
$$

It is easy to check that this function is continuous at $\mu$ and $\gamma$. The problem now is the minimization of this, three branches, function $T C\left(t_{1}\right)$. This requires, separately, studying each of these branches and then combining the results to state the algorithm giving the optimal policy.

\subsection{The Optimal Replenishment Policy}

In this subsection we derive the optimal replenishment policy, that is, we calculate the value, say $t_{1}^{*}$, which minimizes the total cost function. Taking the first-order derivative of $T C_{1}\left(t_{1}\right)$, say $K_{1}\left(t_{1}\right)$, and equating it to zero gives:

$$
\begin{aligned}
K_{1}\left(t_{1}\right)= & -\left(c_{1}+c_{3} \alpha b t_{1}^{b-1}\right) e^{-\alpha t_{1}^{b}}\left[\int_{t_{1}}^{\mu} e^{\alpha x^{b}} f(x) d x+f(\mu) \int_{\mu}^{\gamma} e^{\alpha x^{b}} d x+\int_{\gamma}^{T} e^{\alpha x^{b}} g(x) d x\right] \\
& +\int_{0}^{t_{1}}\left[c_{2} \beta\left(t_{1}-t\right)+c_{2}\left(t_{1}-t\right) \beta^{\prime}\left(t_{1}-t\right)-c_{4} \beta^{\prime}\left(t_{1}-t\right)\right] f(t) d t=0 .
\end{aligned}
$$

If $t_{1}^{*}$ is a root of $(4.21)$, for this root the second-order condition for minimum is

$$
\begin{aligned}
& {\left[c_{1}+c_{3} \alpha b\left(t_{1}^{*}\right)^{b-1}-c_{3}(b-1)\left(t_{1}^{*}\right)^{-1}\right] \alpha b\left(t_{1}^{*}\right)^{b-1} e^{-\alpha t_{1}^{* b}}} \\
& \quad \times\left[\int_{t_{1}^{*}}^{\mu} e^{\alpha x^{b}} f(x) d x+f(\mu) \int_{\mu}^{\gamma} e^{\alpha x^{b}} d x+\int_{\gamma}^{T} e^{\alpha x^{b}} g(x) d x\right] \\
& \quad+\left[c_{1}+c_{3} \alpha b\left(t_{1}^{*}\right)^{b-1}\right] f\left(t_{1}^{*}\right)+\left[c_{2}-c_{4} \beta^{\prime}(0)\right] f\left(t_{1}^{*}\right) \\
& \quad+\int_{0}^{t_{1}^{*}}\left[2 c_{2} \beta^{\prime}\left(t_{1}^{*}-t\right)+c_{2}\left(t_{1}^{*}-t\right) \beta^{\prime \prime}\left(t_{1}^{*}-t\right)-c_{4} \beta^{\prime \prime}\left(t_{1}^{*}-t\right)\right] f(t) d t>0 .
\end{aligned}
$$

So, if (4.22) holds and $t_{1}^{*} \leq \mu$, then the value of order level, $S$, is

$$
S^{*}=I\left(t_{1}^{*}\right)=e^{-\alpha t_{1}^{* b}}\left[\int_{t_{1}^{*}}^{\mu} e^{\alpha x^{b}} f(x) d x+f(\mu) \int_{\mu}^{\gamma} e^{\alpha x^{b}} d x+\int_{\gamma}^{T} e^{\alpha x^{b}} g(x) d x\right],
$$


the ordering quantity is

$$
Q^{*}=\int_{0}^{t_{1}^{*}} f(x) \beta\left(t_{1}^{*}-x\right) d x+S^{*},
$$

and the total cost is $T C_{1}\left(t_{1}^{*}\right)$.

Equating the first-order derivative of $T C_{2}\left(t_{1}\right)$, say $K_{2}\left(t_{1}\right)$, to zero gives

$$
\begin{aligned}
K_{2}\left(t_{1}\right)= & -\left(c_{1}+c_{3} \alpha b t_{1}^{b-1}\right) e^{-\alpha t_{1}^{b}}\left(f(\mu) \int_{t_{1}}^{\gamma} e^{\alpha x^{b}} d x+\int_{\gamma}^{T} e^{\alpha x^{b}} g(x) d x\right) \\
& +\int_{0}^{\mu}\left[c_{2} \beta\left(t_{1}-t\right)+c_{2}\left(t_{1}-t\right) \beta^{\prime}\left(t_{1}-t\right)-c_{4} \beta^{\prime}\left(t_{1}-t\right)\right] f(t) d t \\
& +f(\mu) \int_{\mu}^{t_{1}}\left[c_{2} \beta\left(t_{1}-t\right)+c_{2}\left(t_{1}-t\right) \beta^{\prime}\left(t_{1}-t\right)-c_{4} \beta^{\prime}\left(t_{1}-t\right)\right] d t=0 .
\end{aligned}
$$

If $t_{1}^{*}$ is a root of (4.25), for this root the second-order condition for minimum is

$$
\begin{aligned}
& {\left[c_{1}+c_{3} \alpha b t_{1}^{*^{b-1}}\right] \alpha b t_{1}^{*^{b-1}} e^{-\alpha\left(t_{1}^{*}\right)^{b}}\left(f(\mu) \int_{t_{1}^{*}}^{\gamma} e^{\alpha x^{b}} d x+\int_{\gamma}^{T} e^{\alpha x^{b}} g(x) d x\right)} \\
& \quad-c_{3} \alpha b(b-1) t_{1}^{*(b-2)} e^{-\alpha\left(t_{1}^{*}\right)^{b}}\left(f(\mu) \int_{t_{1}^{*}}^{\gamma} e^{\alpha x^{b}} d x+\int_{\gamma}^{T} e^{\alpha x^{b}} g(x) d x\right)+\left[c_{1}+c_{3} \alpha b\left(t_{1}^{*}\right)^{b-1}\right] f(\mu) \\
& \quad+f(\mu)\left(c_{2}-c_{4} \beta^{\prime}(0)\right)+\int_{0}^{\mu}\left[2 c_{2} \beta^{\prime}\left(t_{1}^{*}-t\right)+c_{2}\left(t_{1}^{*}-t\right) \beta^{\prime \prime}\left(t_{1}^{*}-t\right)-c_{4} \beta^{\prime \prime}\left(t_{1}^{*}-t\right)\right] f(t) d t \\
& \quad+f(\mu) \int_{\mu}^{t_{1}^{*}}\left[2 c_{2} \beta^{\prime}\left(t_{1}^{*}-t\right)+c_{2}\left(t_{1}^{*}-t\right) \beta^{\prime \prime}\left(t_{1}^{*}-t\right)-c_{4} \beta^{\prime \prime}\left(t_{1}^{*}-t\right)\right] d t>0 .
\end{aligned}
$$

So, if (4.26) holds and $\mu<t_{1}^{*}<\gamma$, then the value of $S$ is

$$
S^{*}=I\left(t_{1}^{*}\right)=e^{-\alpha t_{1}^{* b}}\left[f(\mu) \int_{t_{1}^{*}}^{\gamma} e^{\alpha x^{b}} d x+\int_{\gamma}^{T} e^{\alpha x^{b}} g(x) d x\right] .
$$

the ordering quantity is

$$
Q^{*}=\int_{0}^{\mu} f(x) \beta\left(t_{1}^{*}-x\right) d x+f(\mu) \int_{\mu}^{t_{1}^{*}} \beta\left(t_{1}^{*}-x\right) d x+S^{*},
$$

and the total cost is $T C_{2}\left(t_{1}^{*}\right)$. 
Equating the first-order derivative of $T C_{3}\left(t_{1}\right)$, say $K_{3}\left(t_{1}\right)$, to zero gives

$$
\begin{aligned}
K_{3}\left(t_{1}\right)= & -\left(c_{1}+c_{3} \alpha b t_{1}^{b-1}\right) e^{-\alpha t_{1}^{b}} \int_{t_{1}}^{T} e^{\alpha x^{b}} g(x) d x \\
& +\int_{0}^{\mu}\left[c_{2} \beta\left(t_{1}-t\right)+c_{2}\left(t_{1}-t\right) \beta^{\prime}\left(t_{1}-t\right)-c_{4} \beta^{\prime}\left(t_{1}-t\right)\right] f(t) d t \\
& +f(\mu) \int_{\mu}^{\gamma}\left[c_{2} \beta\left(t_{1}-t\right)+c_{2}\left(t_{1}-t\right) \beta^{\prime}\left(t_{1}-t\right)-c_{4} \beta^{\prime}\left(t_{1}-t\right)\right] d t \\
& +\int_{\gamma}^{t_{1}}\left[c_{2} \beta\left(t_{1}-t\right)+c_{2}\left(t_{1}-t\right) \beta^{\prime}\left(t_{1}-t\right)-c_{4} \beta^{\prime}\left(t_{1}-t\right)\right] g(t) d t=0 .
\end{aligned}
$$

If $t_{1}^{*}$ is a root of (4.29), for this root the second-order condition for minimum is

$$
\begin{aligned}
& {\left[c_{1}+c_{3} \alpha b t_{1}^{*^{b-1}}\right] \alpha b t_{1}^{k^{b-1}} e^{-\alpha\left(t_{1}^{*}\right)^{b}} \int_{t_{1}^{*}}^{T} e^{\alpha x^{b}} g(x) d x} \\
& -c_{3} \alpha b(b-1) t_{1}^{(b-2)} e^{-\alpha\left(t_{1}^{*}\right)^{b}} \int_{t_{1}^{*}}^{T} e^{\alpha x^{b}} g(x) d x+\left[c_{1}+c_{3} \alpha b\left(t_{1}^{*}\right)^{b-1}\right] g\left(t_{1}^{*}\right) \\
& \quad+g\left(t_{1}^{*}\right)\left(c_{2}-c_{4} \beta^{\prime}(0)\right)+\int_{0}^{\mu}\left[2 c_{2} \beta^{\prime}\left(t_{1}^{*}-t\right)+c_{2}\left(t_{1}^{*}-t\right) \beta^{\prime \prime}\left(t_{1}^{*}-t\right)-c_{4} \beta^{\prime \prime}\left(t_{1}^{*}-t\right)\right] f(t) d t \\
& +f(\mu) \int_{\mu}^{\gamma}\left[2 c_{2} \beta^{\prime}\left(t_{1}^{*}-t\right)+c_{2}\left(t_{1}^{*}-t\right) \beta^{\prime \prime}\left(t_{1}^{*}-t\right)-c_{4} \beta^{\prime \prime}\left(t_{1}^{*}-t\right)\right] d t \\
& +\int_{\gamma}^{t_{1}^{*}}\left[2 c_{2} \beta^{\prime}\left(t_{1}^{*}-t\right)+c_{2}\left(t_{1}^{*}-t\right) \beta^{\prime \prime}\left(t_{1}^{*}-t\right)-c_{4} \beta^{\prime \prime}\left(t_{1}^{*}-t\right)\right] g(t) d t>0 .
\end{aligned}
$$

So, if (4.30) holds and $t_{1}^{*}>\gamma$, then the value of $S$ is

$$
S^{*}=I\left(t_{1}^{*}\right)=e^{-a\left(t_{1}^{*}\right)^{b}} \int_{t_{1}^{*}}^{T} e^{a x^{b}} g(x) d x
$$

the ordering quantity is

$$
Q^{*}=\int_{0}^{\mu} f(x) \beta\left(t_{1}^{*}-x\right) d x+f(\mu) \int_{\mu}^{\gamma} \beta\left(t_{1}^{*}-x\right) d x+\int_{\gamma}^{t_{1}^{*}} g(x) \beta\left(t_{1}^{*}-x\right) d x+S^{*},
$$

and the total cost is $T C_{3}\left(t_{1}^{*}\right)$.

Remark 4.1. Due to (4.21), (4.25), and (4.29) the function $T C\left(t_{1}\right)$ is differentiable at the point $\mu$ and $\gamma$. 
In the previous analysis there is no guarantee that $t_{1}^{*}$ exists and corresponds to the minimum. Its uniqueness is also another issue. The proposition, which follows, provides sufficient conditions for existence, uniqueness, and validity of $t_{1}^{*}$.

Let us set

$$
\begin{aligned}
\Delta_{1}= & K_{1}(\mu) \\
= & -\left(c_{1}+c_{3} \alpha b \mu^{b-1}\right) e^{-\alpha \mu^{b}}\left[f(\mu) \int_{\mu}^{\gamma} e^{\alpha x^{b}} d x+\int_{\gamma}^{T} e^{\alpha x^{b}} g(x) d x\right] \\
& +\int_{0}^{\mu}\left[c_{2} \beta(\mu-t)+c_{2}(\mu-t) \beta^{\prime}(\mu-t)-c_{4} \beta^{\prime}(\mu-t)\right] f(t) d t, \\
\Delta_{2}= & K_{2}(\gamma) \\
= & -\left(c_{1}+c_{3} \alpha b \gamma^{b-1}\right) e^{-\alpha \gamma^{b}}\left(\int_{\gamma}^{T} e^{\alpha x^{b}} g(x) d x\right) \\
& +\int_{0}^{\mu}\left[c_{2} \beta(\gamma-t)+c_{2}(\gamma-t) \beta^{\prime}(\gamma-t)-c_{4} \beta^{\prime}(\gamma-t)\right] f(t) d t \\
& +f(\mu) \int_{\mu}^{\gamma}\left[c_{2} \beta(\gamma-t)+c_{2}(\gamma-t) \beta^{\prime}(\gamma-t)-c_{4} \beta^{\prime}(\gamma-t)\right] d t
\end{aligned}
$$

and $h(t)=c_{2} \beta(t)+c_{2} t \beta^{\prime}(t)-c_{4} \beta^{\prime}(t)$.

The following proposition can be easily proved observing that $K_{1}(0)<0$ and $K_{3}(T)>0$.

Proposition 4.2. If $b<1, h^{\prime}(t)>0, t \in[0, T]$ and

(1) $\Delta_{1}>0$, then (4.21) has one root, say $t_{1}^{*}$, which is the unique optimal value of the problem.

(2) $\Delta_{1}<0$, and $\Delta_{2}>0$ then (4.25) has one root, say $t_{1}^{*}$, which is the unique optimal value of the problem.

(3) $\Delta_{1}<0$, and $\Delta_{2}<0$ then (4.29) has one root, say $t_{1}^{*}$, which is the unique optimal value of the problem.

We note that the above proposition ensures the existence, uniqueness, and validity of $t_{1}$. If the conditions of this proposition do not hold, then the following procedure can be used to calculate the optimal replenishment policy.

Step 1. (1) Find the global minimizing point, $t_{1}^{*}$, for $T C_{1}\left(t_{1}\right)$. This will be one of the following points:

(a) root of $(4.21)$, (an interior point of $[0, \mu])$ which satisfies $(4.22)$

(b) $t_{1}^{*}=0$,

(c) $t_{1}^{*}=\mu$.

Then calculate $T C_{1}\left(t_{1}^{*}\right)$. 
(2) Find the global minimizing, $t_{1}^{*}$, for $T C_{2}\left(t_{1}\right)$. This will be one of the following points:

(a) a root of (4.25), (an interior point of $[\mu, T]$ ) which satisfies (4.26),

(b) $t_{1}^{*}=\mu$,

(c) $t_{1}^{*}=\gamma$.

Then calculate $T C_{2}\left(t_{1}^{*}\right)$.

(3) Find the global minimizing, $t_{1}^{*}$, for $T C_{3}\left(t_{1}\right)$. This will be one of the following points:

(a) a root of (4.29), (an interior point of $[\mu, T]$ ) which satisfies (4.30),

(b) $t_{1}^{*}=\gamma$,

(c) $t_{1}^{*}=\mathrm{T}$.

Then calculate $T C_{3}\left(t_{1}^{*}\right)$.

Step 2. Find the $\min \left\{T C_{1}\left(t_{1}^{*}\right), T C_{2}\left(t_{1}^{*}\right), T C_{3}\left(t_{1}^{*}\right)\right\}$ and accordingly select the optimum $t_{1}^{*}$.

Remark 4.3. The analysis shows that, in this model, $t_{1}^{*}$ is dependent from the demand rate $D(t)$.

\section{Numerical Examples}

The examples, which follow, illustrate the results obtained.

Example 5.1. The input parameters are $c_{1}=\$ 3$ per unit per year, $c_{2}=\$ 15$ per unit per year, $c_{3}=\$ 5$ per unit, $c_{4}=\$ 20$ per unit, $\mu=0.12$ year, $\gamma=0.9, a=0.001, b=2, T=1$ year, $f(t)=3 e^{4.5 t}, g(t)=3 e^{4.5 \mu-0.8(t-\gamma)}$, and $\beta(x)=e^{-0.2 x}$.

Model Starting with No Shortages

Using (3.37) the optimal value of $t_{1}$ is $\mu<t_{1}^{*}=0.860<\gamma$, and consequently the optimal ordering quantity is $Q^{*}=4.98$ (from (3.39)) and the minimum cost is $T C\left(t_{1}^{*}\right)=6.631$ (from $(3.23))$.

\section{Model Starting with Shortages}

Using (4.25) the optimal value of $t_{1}$ is $\mu<t_{1}^{*}=0.163<\gamma$, and consequently the optimal ordering quantity is $Q^{*}=4.98$ (from (4.28)), and the minimum cost is $T C\left(t_{1}^{*}\right)=6.329$ (from (4.13)).

Example 5.2. This example is identical to Example 5.1, except that $\gamma=0.7, f(t)=20 e^{3.5 t}$, and $g(t)=20 e^{3.5 \mu}-50(t-\gamma)$.

\section{Model Starting with No Shortages}

Again $t_{1}^{*}=0.860$ but now $\gamma<t_{1}^{*}$, the optimal ordering quantity is $Q^{*}=27.49$ (from (3.40)), and the minimum cost is $T C\left(t_{1}^{*}\right)=35.855$ (from (3.34)). 
Table 1: Sensitivity analysis for model starting without shortages.

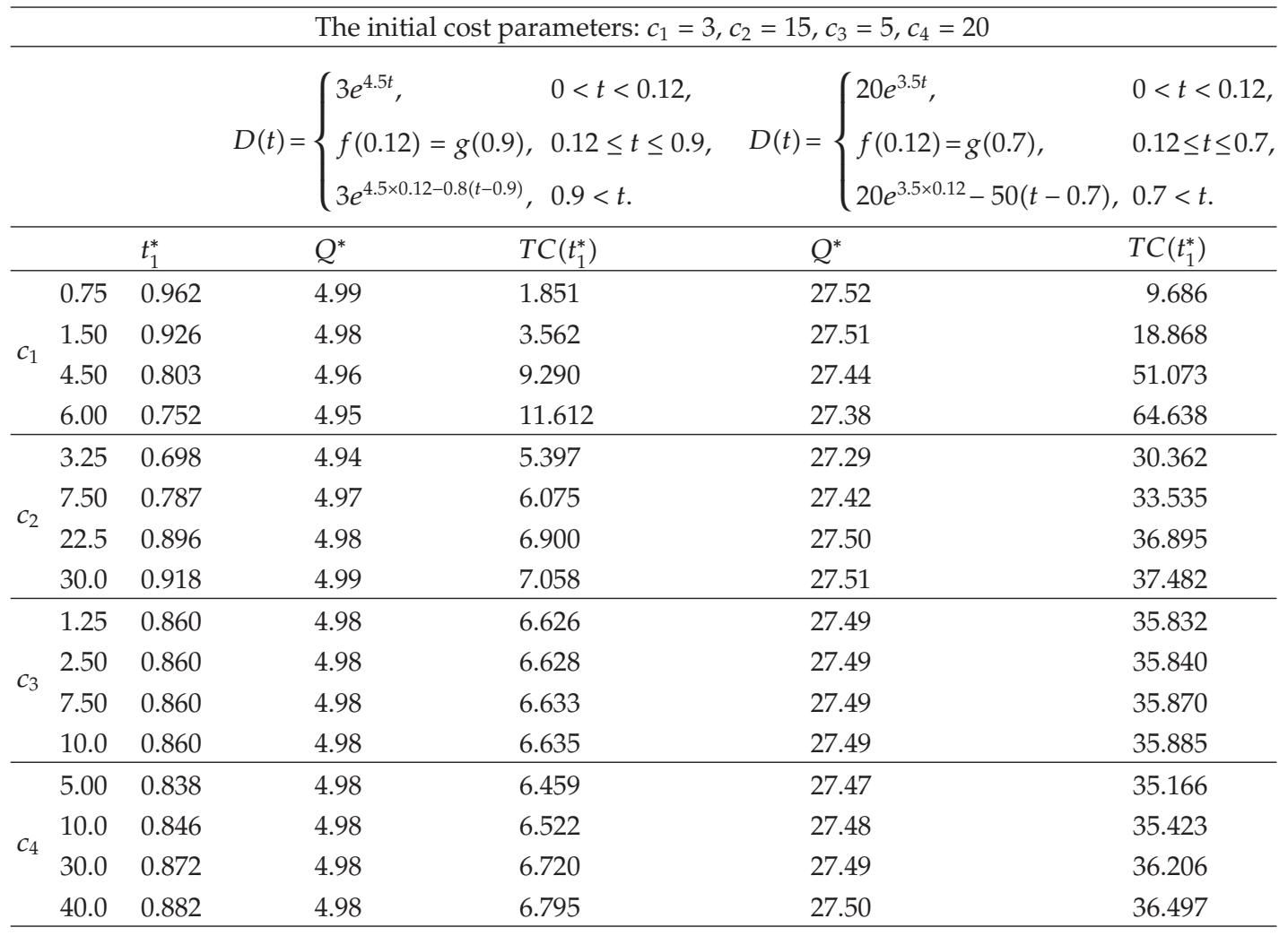

\section{Model Starting with Shortages}

Using (4.25) the optimal value of $t_{1}$ is $\mu<t_{1}^{*}=0.148<\gamma$, and consequently the optimal ordering quantity is $Q^{*}=27.48$ (from (4.28)) and the minimum cost is $T C\left(t_{1}^{*}\right)=33.008$ (from (4.13)).

In Tables 1 and 2 some sensitivity analysis for the models starting without and with shortages, respectively, is performed, for the above examples, by changing the cost parameter values $-75 \%,-50 \%,+50 \%$ and,$+100 \%$ taking one at a time and keeping the remaining unchanged. From these two examples and the sensitivity analysis is evident the following.

(1) For the model starting without shortages, although the time when shortages occur $\left(t_{1}^{*}\right)$ is identical for the two examples, the ordering quantities and costs are significant different, obviously because of the demand rate.

(2) For the two models the changes in the total optimal costs indicate that the models are highly sensitive to the error on the estimation of the parameter value $c_{1}$, moderately sensitive to the error on $c_{2}$, while low sensitivity is to the error on the estimation of the parameters $c_{3}$ and $c_{4}$. In presented examples, the costs related to storage inventory $\left(c_{1}\right.$ and $\left.c_{3}\right)$ are less than costs related to unsatisfied demand $\left(c_{2}\right.$ and $\left.c_{4}\right)$ maybe this explains the low sensitivity to the error on the estimation of the parameter $c_{4}$. While the small deterioration rate maybe implies low sensitivity with respect to $c_{3}$. 
Table 2: Sensitivity analysis for model starting with shortages.

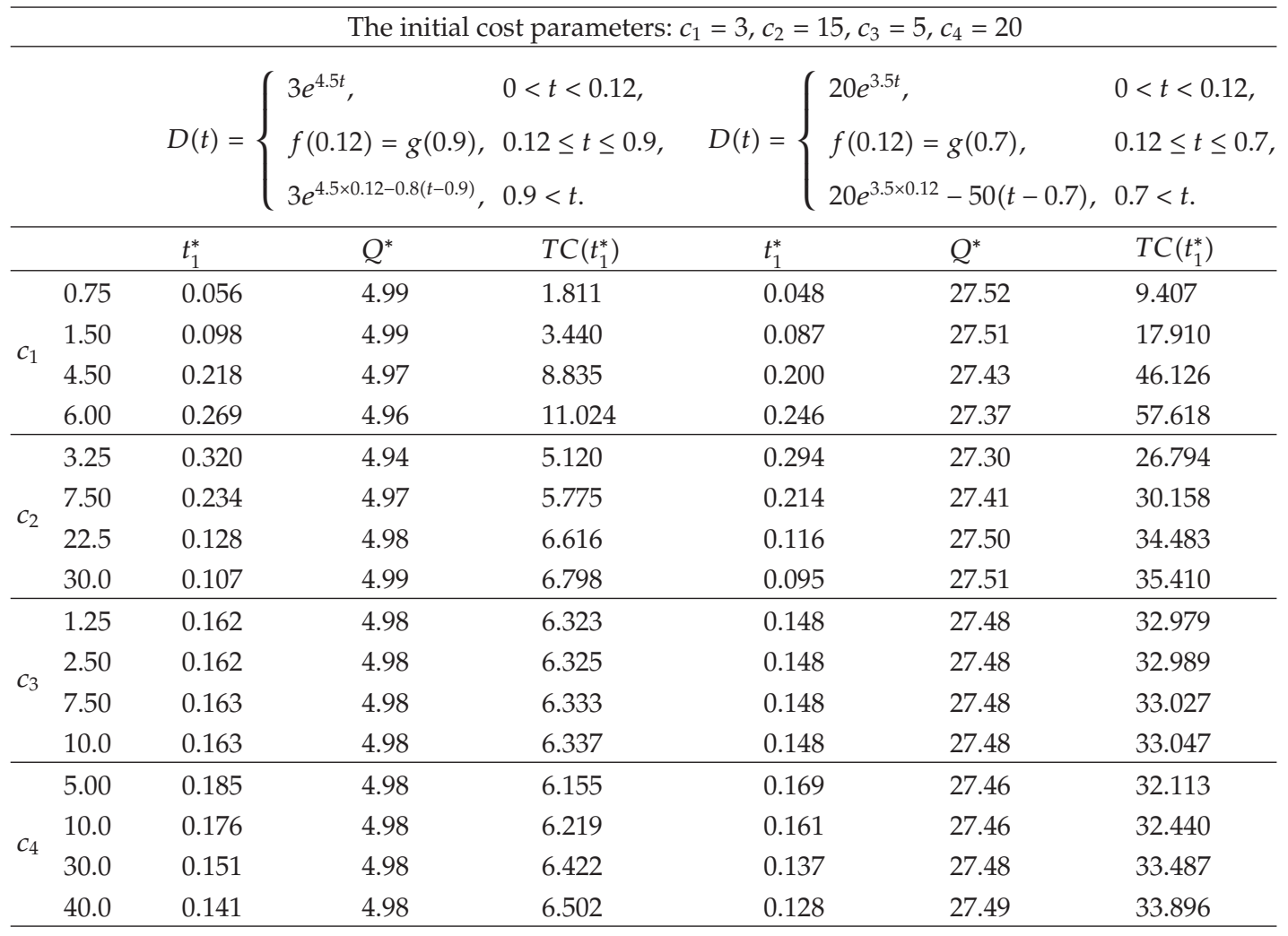

(3) The errors in cost parameters are attenuated when translated into changes in the optimal ordering quantity.

\section{Concluding Remarks}

In this paper, an order level inventory model for deteriorating items has been studied. The basic assumption of the model is based on time dependent three branches ramp type demand rate. The demand of seasonable and fashionable products can be described well with this function, as the nature of demand of these products is increasing at the beginning of the season, steady in the mid of the season, and decreasing at the end of the season. To the best of our knowledge this demand pattern studied for the first time, at least, using so general functions for the nonsteady periods. In addition a time dependent backlogging and deterioration rate are assumed. The inventory model is studied under two different replenishment policies: (a) starting with no shortages and (b) starting with shortages. An algorithm to obtain the optimal policy is proposed.

Moreover from this model follows as special cases the following ones.

(1) If $f(t)=D_{0} t, t \in[0, \mu], g(x)=f(\mu), \mu=\gamma$ and $\beta(x)=1 /(1+\delta x)$, this model reduces to that of $\mathrm{Wu}[12]$.

(2) If in addition to (1), $\beta(x)=1$ (case of complete backlogging), then it further reduces to that of Wu et al. [10]. 
(3) If $b=1$ (i.e., constant deterioration rate), $f(t)=D_{0} t, g(x)=f(\mu), \mu=\gamma$, and $\beta(x)=1$, then the models reduce to those of Wu and Ouyang [11], Mandal and Pal [9], and Deng et al. [15].

(4) If $g(x)=f(\mu), \mu=\gamma$, this model gives the one model proposed by Skouri et al. [16].

\section{Acknowledgment}

The authors would like to thank the referees for their valuable comments and suggestions that improved the paper.

\section{References}

[1] M. Resh, M. Friedman, and L. C. Barbosa, "On a general solution of the deterministic lot size problem with time-proportional demand," Operations Research, vol. 24, no. 4, pp. 718-725, 1976.

[2] W. A. Donaldson, "Inventory replenishment policy for a linear trend in demand: an analytic solution," Operational Research Quarterly, vol. 28, pp. 663-670, 1977.

[3] U. Dave and L. K. Patel, “ $\left(T, \mathrm{~S}_{\mathrm{i}}\right)$ policy inventory model for deteriorating items with time proportional demand," Journal of the Operational Research Society, vol. 32, no. 2, pp. 137-142, 1981.

[4] S. K. Goyal, "On improving replenishment policies for linear trend in demand," Engineering Costs and Production Economics, vol. 10, no. 1, pp. 73-76, 1986.

[5] M. Hariga, "An EOQ model for deteriorating items with shortages and time varying demand," Journal of the Operational Research Society, vol. 46, pp. 398-404, 1995.

[6] M. A. Hariga and L. Benkherouf, "Optimal and heuristic inventory replenishment models for deteriorating items with exponential time-varying demand," European Journal of Operational Research, vol. 79, no. 1, pp. 123-137, 1994.

[7] H.-L. Yang, J.-T. Teng, and M.-S. Chern, “Deterministic inventory lot-size models under inflation with shortages and deterioration for fluctuating demand," Naval Research Logistics, vol. 48, no. 2, pp. 144 158, 2001.

[8] R. M. Hill, "Inventory models for increasing demand followed by level demand," Journal of the Operational Research Society, vol. 46, no. 10, pp. 1250-1259, 1995.

[9] B. Mandal and A. K. Pal, "Order level inventory system with ramp type demand rate for deteriorating items," Journal of Interdisciplinary Mathematics, vol. 1, no. 1, pp. 49-66, 1998.

[10] J.-W. Wu, C. Lin, B. Tan, and W.-C. Lee, "An EOQ inventory model with ramp type demand rate for items with Weibull deterioration," International Journal of Information and Management Sciences, vol. 10, no. 3, pp. 41-51, 1999.

[11] K.-S. Wu and L.-Y. Ouyang, "A replenishment policy for deteriorating items with ramp type demand rate," Proceedings of the National Science Council, Republic of China A, vol. 24, no. 4, pp. 279-286, 2000.

[12] K.-S. Wu, "An EOQ inventory model for items with Weibull distribution deterioration, ramp type demand rate and partial backlogging," Production Planning and Control, vol. 12, no. 8, pp. 787-793, 2001.

[13] B. C. Giri, A. K. Jalan, and K. S. Chaudhuri, "Economic order quantity model with Weibull deterioration distribution, shortage and ramp-type demand," International Journal of Systems Science, vol. 34, no. 4, pp. 237-243, 2003.

[14] S. K. Manna and K. S. Chaudhuri, "An EOQ model with ramp type demand rate, time dependent deterioration rate, unit production cost and shortages," European Journal of Operational Research, vol. 171, no. 2, pp. 557-566, 2006.

[15] P. S. Deng, R. H.-J. Lin, and P. Chu, "A note on the inventory models for deteriorating items with ramp type demand rate," European Journal of Operational Research, vol. 178, no. 1, pp. 112-120, 2007.

[16] K. Skouri, I. Konstantaras, S. Papachristos, and I. Ganas, "Inventory models with ramp type demand rate, partial backlogging and Weibull deterioration rate," European Journal of Operational Research, vol. 192, no. 1, pp. 79-92, 2009.

[17] P. M. Ghare and G. F. Schrader, "A model for exponentially decaying inventories," Journal of Industrial Engineering, vol. 14, pp. 238-243, 1963. 
[18] R. P. Covert and G. C. Philip, "An EOQ model for items with Weibull distribution deterioration," AIIE Transaction, vol. 5, no. 4, pp. 323-326, 1973.

[19] P. R. Tadikamalla, "An EOQ inventory model for items with gamma distribution," AIIE Transaction, vol. 10, no. 1, pp. 100-103, 1978.

[20] F. Raafat, "Survey of literature on continuously deteriorating inventory models," Journal of the Operational Research Society, vol. 42, no. 1, pp. 27-37, 1991.

[21] S. K. Goyal and B. C. Giri, "Recent trends in modeling of deteriorating inventory," European Journal of Operational Research, vol. 134, no. 1, pp. 1-16, 2001.

[22] P. L. Abad, "Optimal pricing and lot-sizing under conditions of perishability and partial backordering," Management Science, vol. 42, no. 8, pp. 1093-1104, 1996.

[23] H.-J. Chang and C.-Y. Dye, "An EOQ model for deteriorating items with time varying demand and partial backlogging," Journal of the Operational Research Society, vol. 50, no. 11, pp. 1176-1182, 1999.

[24] K. Skouri and S. Papachristos, "A continuous review inventory model, with deteriorating items, time-varying demand, linear replenishment cost, partially time-varying backlogging," Applied Mathematical Modelling, vol. 26, no. 5, pp. 603-617, 2002.

[25] J.-T. Teng, H.-J. Chang, C.-Y. Dye, and C.-H. Hung, “An optimal replenishment policy for deteriorating items with time-varying demand and partial backlogging," Operations Research Letters, vol. 30, no. 6, pp. 387-393, 2002.

[26] S.-P. Wang, "An inventory replenishment policy for deteriorating items with shortages and partial backlogging," Computers E Operations Research, vol. 29, no. 14, pp. 2043-2051, 2002.

[27] L. A. San José, J. Sicilia, and J. García-Laguna, "An inventory system with partial backlogging modeled according to a linear function," Asia-Pacific Journal of Operational Research, vol. 22, no. 2, pp. 189-209, 2005.

[28] L. A. San José, J. Sicilia, and J. García-Laguna, "Analysis of an inventory system with exponential partial backordering," International Journal of Production Economics, vol. 100, no. 1, pp. 76-86, 2006.

[29] C.-K. Chen, T.-W. Hung, and T.-C. Weng, "Optimal replenishment policies with allowable shortages for a product life cycle," Computers $\mathcal{E}$ Mathematics with Applications, vol. 53, no. 10, pp. 1582-1594, 2007.

[30] C.-K. Chen, T.-W. Hung, and T.-C. Weng, "A net present value approach in developing optimal replenishment policies for a product life cycle," Applied Mathematics and Computation, vol. 184, no. 2, pp. 360-373, 2007.

[31] E. Naddor, Inventory Systems, John Wiley \& Sons, New York, NY, USA, 1966. 


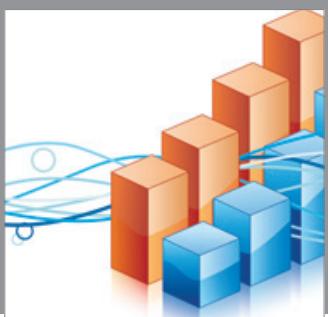

Advances in

Operations Research

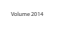

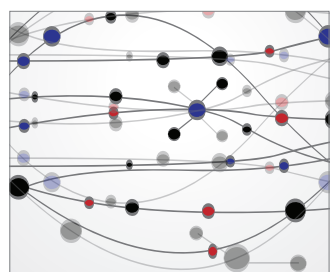

\section{The Scientific} World Journal
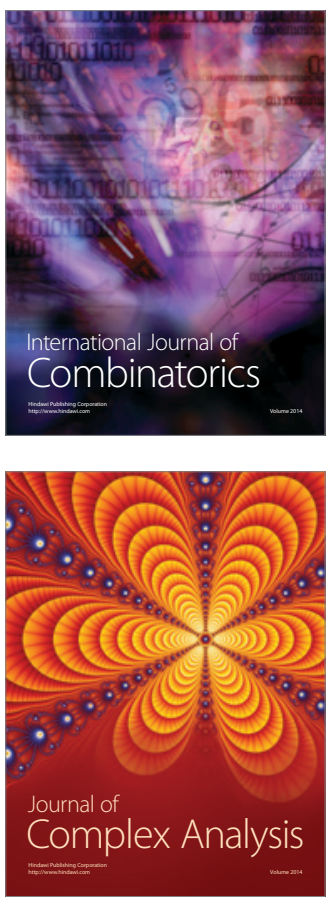

International Journal of

Mathematics and

Mathematical

Sciences
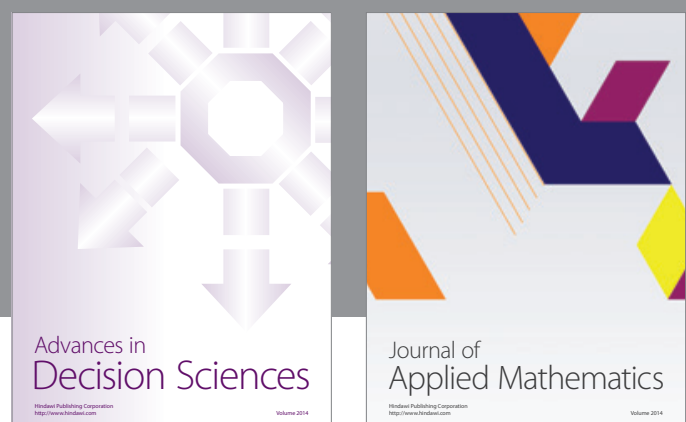

Journal of

Applied Mathematics
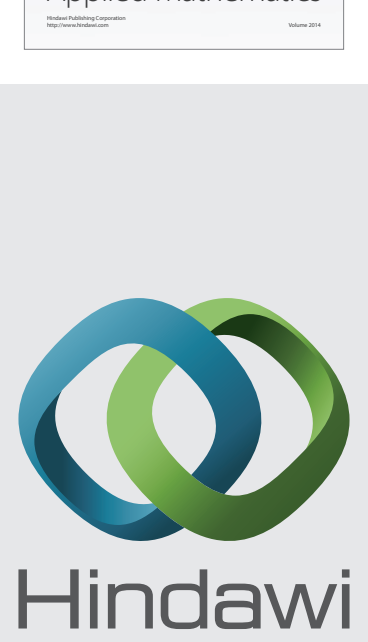

Submit your manuscripts at http://www.hindawi.com
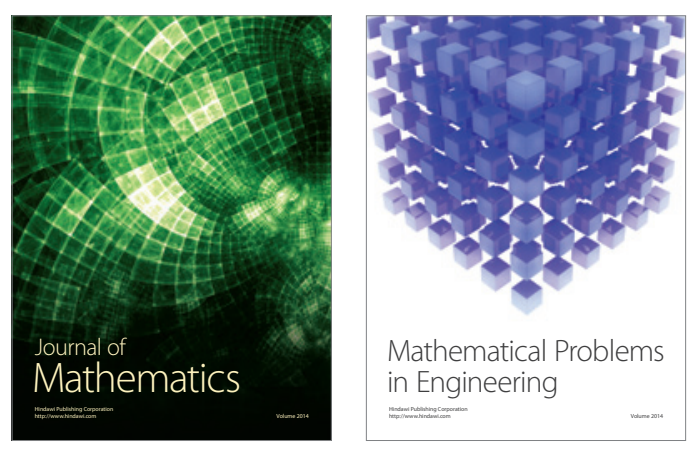

Mathematical Problems in Engineering
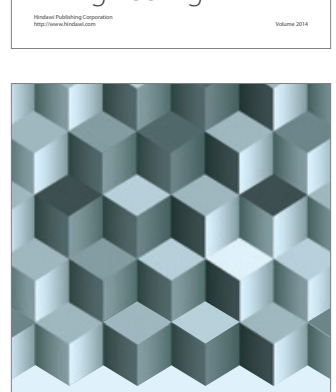

Journal of

Function Spaces
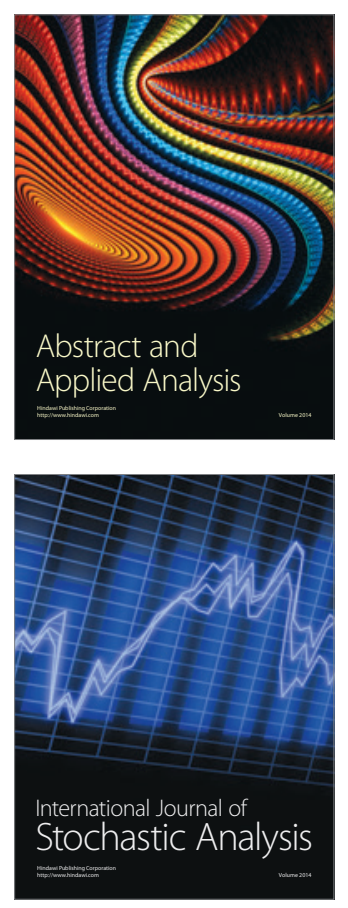

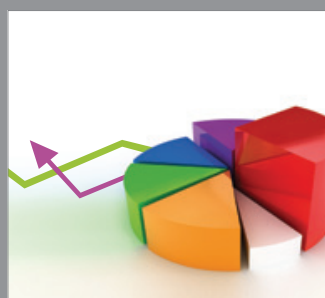

ournal of

Probability and Statistics

Promensencen
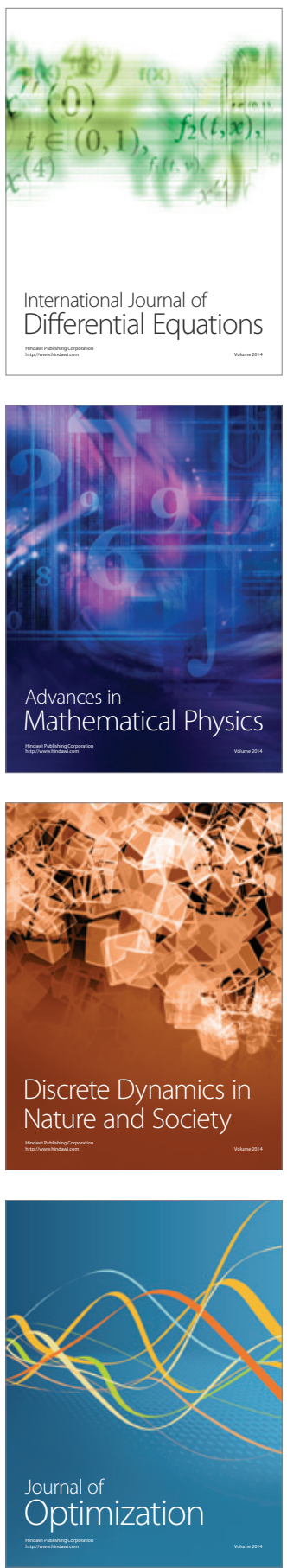